\title{
Case Study: Interacting with Cortical Flat Maps of the Human Brain
}

\author{
Monica K. Hurdal* \\ Department of Mathematics \\ and $\mathrm{CSIT}^{\dagger}$ \\ Florida State University and \\ International Neuroimaging Consortium \\ VA Medical Center \\ University of Minneapolis \\ mhurdal@math.fsu.edu
}

\author{
Kevin W. Kurtz \\ Department of Computer Science \\ and $\mathrm{CSIT}^{\dagger}$ \\ Florida State University \\ kurtz@csit.fsu.edu
}

\author{
David C. Banks \\ Department of Computer Science \\ and $\mathrm{CSIT}^{\dagger}$ \\ Florida State University \\ banks@csit.fsu.edu
}

\begin{abstract}
The complex geometry of the human brain contains many folds and fissures, making it impossible to view the entire surface at once. Since most of the cortical activity occurs on these folds, it is desirable to be able to view the entire surface of the brain in a single view. This can be achieved using quasi-conformal flat maps of the cortical surface. Computational and visualization tools are now needed to be able to interact with these flat maps of the brain to gain information about spatial and functional relationships that might not otherwise be apparent. Such information can contribute to earlier diagnostic tools for diseases and improved treatment. Our group is developing visualization and analysis tools that will help elucidate new information about the human brain through the interaction between a cortical surface and its corresponding quasiconformal flat map.
\end{abstract}

CR Categories: J.3 [Computer Applications]: Life and Medical Sciences; I.3.3 [Computer Graphics]: Picture/Image Generation; I.3.8 [Computer Graphics]: Applications

Keywords: Conformal, Cortical Features, Human Brain, Flat Map, Interaction, MRI, Neuroscience, Surface

\section{Introduction}

The human brain is a highly convoluted organ with many folds and fissures. There is considerable variability and individual differences in the size, location and extent of these fissures and folds across people. The thin layer of the cortical surface - the grey matter or cerebrum - is about $3 \mathrm{~mm}$ thick and is where most of the cortical activity and functional processing of the brain occur. The interior of the brain is largely comprised of white matter which transmits signals and connects cortical regions. The brain can be divided into various lobes and regions based on function and anatomy. For example, there are specific regions devoted to vision (the occipital lobe), smell, hearing and taste (the temporal lobe) and peripheral sensations from the body (the parietal lobe) [7].

The surface area of the cerebral cortex grey matter is approximately $1570 \mathrm{~cm}^{2}$, of which $60-70 \%$ is buried in the folds of

* Address correspondence to Dr. Monica K. Hurdal, Florida State University, Department of Mathematics, Tallahassee, FL, 32306-4510. Ph: (850) 644-7378; Fax: (850) 644-4053; Email: mhurdal@math.fsu.edu; URL: http://www.math.fsu.edu/ mhurdal

${ }^{\dagger}$ School of Computational Science and Information Technology
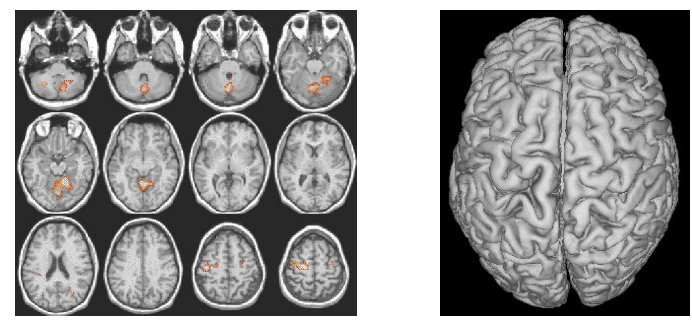

Figure 1: MRI Brain Slices and an Isosurface of the Cerebrum.

the brain and hidden from view [17]. The surface is topologically equivalent to a cortical sheet. Non-invasive anatomical data is obtained from magnetic resonance imaging (MRI) scans and functional data is obtained from a variety of modalities including positron emission tomography (PET), functional MRI (fMRI), electroencephalography (EEG) and magnetoencephalograpy (MEG). See [16] for a compilation of various methods.

Traditionally, brain data is viewed as a series of single MRI slices with functional activity imposed, as a volume rendering of the reconstructed cortical surface or as an isosurface (see Figure 1). However, these methods are inadequate for viewing functional activity buried in the cortical folds. Flat maps of the cortical surface are being used to address this deficiency $[3,4,17]$.

A member of our group is also a member of the International Neuroimaging Consortium that is based at the VA Medical Center at the University of Minneapolis [6] which is funded by the National Institutes of Health as part of the Human Brain Project and neuroinformatics initiatives [14]. Hurdal has developed a cortical flat mapping tool that is being used by neurologists and researchers to create quasi-conformal flat maps of the brain. Computational and visualization tools are now needed to be able to interact with these flat maps of the brain to gain information about spatial and functional relationships that might not otherwise be apparent. Our group is developing visualization and analysis tools that will help elucidate new information about the human brain through the interaction between the cortical surface in 3-space and the 2D flat map. Although the pipeline from research to clinical practice will take several years, these interactive visualizations will assist neuroscientific researchers in understanding the structure and function of the human brain.

\section{Flat Mapping the Human Brain}

The highly folded cortical surface makes it difficult to visualize and understand functional and spatial relationships of the brain and 
quantify anatomical variability. "Unfolding" the cortical surface and creating flat maps that are readily mapped to the original surface of the brain are important tools for aiding in the understanding of the organization and function of the brain.

The cortical surface of the brain is topologically equivalent to a 2-sphere (i.e. a closed, simply-connected surface with no holes or handles and has an Euler characteristic of 2). Commonly, each hemisphere of the brain is used separately for creating flat maps. A single closed boundary cut is introduced where the hemispheres were separated along the corpus callosum to act as the map boundary under flattening.

A topologically correct, tessellated mesh representing the grey matter surface of the brain is required for flattening. Creating such a surface requires considerable processing of the MRI data. After an MRI volume is acquired, inhomogeneities from the scan must be removed [12]. Then undesired regions (such as the scalp and skull) must be removed or stripped away, leaving a volume containing the cerebral cortex. This new volume can be parcellated into anatomical regions for identification purposes [11] and this information can also be transferred to any flat map that is created. The volume is segmented into various tissue types, including grey matter, white matter and cerebrospinal fluid [9]. An isosurface of the grey matter is created using, for example, a variant of the marching cubes algorithm [8]. Topological problems such as holes, handles and fins must then be removed, resulting in a topologically correct 2-manifold.

It is impossible to flatten a surface with intrinsic curvature (such as the brain) without introducing metric or areal distortions (i.e. a surface with non-constant Gaussian curvature can not be mapped isometrically to a surface with constant Gaussian curvature). However, the Riemann Mapping Theorem of 1852 [1] from complex function theory indicates that it is possible to preserve conformal (angular) information under flattening. This theorem is an existence theorem and until recently it was impossible to compute the conformal map for surfaces as complicated as the brain. Hurdal [4] has adapted a method that uses circle packings to compute an approximation to the conformal map of a cortical surface.

A circle packing is a pattern of circles with a specified pattern of tangencies. More specifically, we can describe our triangulated mesh, $S$, in terms of its combinatorics $K$ and its geometric realization $V$, where $K$ is a (abstract) simplicial 2-complex representing the connectivity of the vertices, edges and faces and $V$ is a set of vertex positions (points in $\mathbb{R}^{3}$ ) defining the shape of the mesh. At each vertex $v$ of $K$ is placed a circle $c_{v}$ so that $c_{v}$ is tangent to $c_{u}$ when $\langle v, u\rangle$ is an edge of $K$ and the triple $\left\langle c_{v}, c_{u}, c_{w}\right\rangle$ of mutually tangent circles is positively oriented in $S$ whenever $\langle v, u, w\rangle$ is a positively oriented face of $K$. This collection of circles is a circle packing and gives us a new surface in $\mathbb{R}^{2}$ which is our flat mapping of $S$ (see Figure 2).

A circle packing is computed using an iterative process that calculates the circle radius for each circle $c_{v}$. Initial radii values for each vertex are computed based on metric information of $S$. Any interior vertex $v$ of $S$ has a closed chain of contiguous neighboring
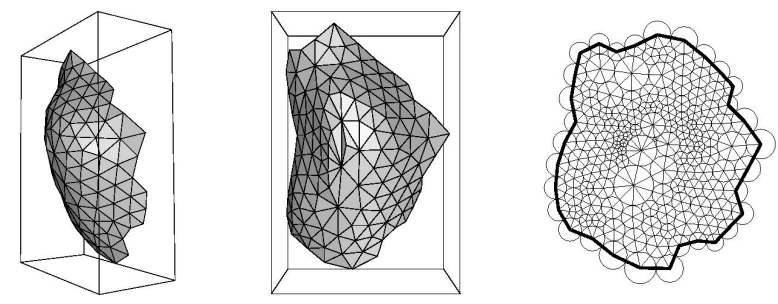

Figure 2: A Surface and its Circle Packing. vertices that form the triangular faces surrounding $v$. The surface at a vertex $v$ can be "flattened" if the angles formed at $v$ from its triangles sum to $2 \pi$. Trigonometry provides a packing condition for flatness at interior $v$ using the radii of $c_{v}$ and its neighbors. The radii of the interior circles are adjusted until the packing condition is satisfied for all interior $v$. Typically there is no packing condition for boundary vertices, which accounts for the extra degrees of freedom a boundary provides.

The circle packing gives the radii of the circles and it is easy compute the location of the circle centers (i.e. vertices) in $\mathbb{R}^{2}$ once the first two tangent circles are laid out. We know that circle packings exist and these circle packing mappings are quasi-conformal, meaning that there is but a bounded amount of percentage angular distortion that depends only on the maximum degree of vertices [2], [10]. There is a comprehensive theory guaranteeing we can compute a circle packing and that it converges to the unique circle packing. It should be noted that the method for computing the circle packing is purely numeric, not geometric, meaning that the circle packing does not exist on the original surface $S$ in $3 D$ and there is no coherent circle packing (i.e. flat map) possible until a sufficiently accurate approximation of the final packing (i.e. flat map) is reached. More details can be found in [5] and [13].

This circle packing approach offers a number of advantages over other flattening schemes. Circle packing provides a theoretical framework (i.e. a mathematical characterization) for computing a first approximation to a conformal map, as well as a flat map. These maps are quasi-conformal and guaranteed to be mathematically unique. In contrast to isometric flat maps, we have the guaranteed existence of conformal flat maps. Maps can be displayed in three geometries: the Euclidean and hyperbolic planes and on a sphere. Conformal maps offer a wide variety of useful manipulations, including Möbius transformations which can be used to zoom and focus the maps in a particular region of interest. A single closed boundary cut is introduced where the cortical surface is isolated from other neural tissues, such as along the brain stem or corpus collosum. Other extraneous cuts are not required. Conformal maps preserve angle proportion. Hence subtle shape information is preserved and this could play a role in cortical studies. Examples of flat maps from the human cerebral cortex are shown in Figure 3 (and its corresponding color plate).
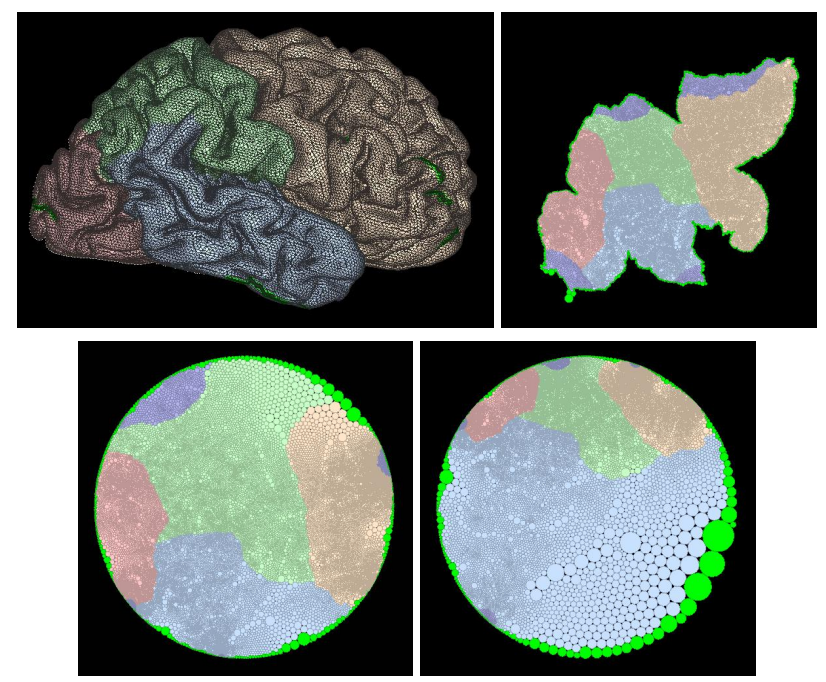

Figure 3: The Human Cerebral Cortex. Top left: the cerebrum; top right: Euclidean flat map; bottom left: hyperbolic flat map; bottom right: hyperbolic map with transformation applied. Colors correspond to various anatomical lobes (see color plate). 


\section{Interacting with Flat Maps}

\subsection{Morphing the Cortical Surface onto the Flat Map}

As indicated in the previous section, there are no intermediate cortical maps produced in the flattening process. The circle packing approach yields a single, final flat map in $\mathbb{R}^{2}$ which is a quasiconformal map of the cortical surface. Recall that each circle in the flat map corresponds to a vertex in the cortical surface mesh in $\mathbb{R}^{3}$. Significant information, such as anatomical labeling or curvature, can be transferred from the original surface to the flattened surface via this correspondence.

It is instructive to be able to understand how the original cortical surface is morphed onto one of our flat map target geometries (i.e. the Euclidean plane, the hyperbolic plane or sphere). Our group used Open Inventor to interactively morph between the original cortical surface mesh in $\mathbb{R}^{3}$ and the precomputed flat map in $\mathbb{R}^{2}$. We used a surface corresponding to the cerebral cortex of the "Visible Man" from The National Library of Medicine's Visible Human Project [15]. This surface consists of 103,845 triangles and 52,360 vertices (see Figure 3 and its corresponding color plate). For this data, a boundary cut along the corpus collosum and four extraneous cuts along various fissures have been introduced to create a surface boundary. Although these extraneous cuts are not needed for the circle packing approach, they are of interest to other neuroscientific researchers [17].

A texture map of the circle packing flat map was imposed on the original cortical surface. This serves the additional purpose of being able to observe the circle deformations that occur when the circles from the circle packing flat map are taken back onto the original surface. The textured circle map is generated from the circle packing using a two dimensional tent filter that spreads points across a given circle radius from the circle packing flat map at the appropriate location. The circle locations are normalized and the circle packing guarantees that each circle corresponds one to one to a vertex in the $3 \mathrm{D}$ mesh.

Linear interpolation was used to compute the morphing that takes the cortical surface onto the precomputed flat map. The user manipulates a $3 \mathrm{D}$ widget within the program to control the morphing. The morphing variable can range from 0.0 (flat map) to 1.0 (3D mesh). Linear interpolation takes each circle in the flat map and interpolates it to its final location in 3D based on the morphing variable. A movie from a user session is provided and some frames are illustrated in Figure 4 (and its corresponding color plate). Again, it should be emphasized that the quasi-conformal map is not computed via this morphing process - a different morphing algorithm will produce different intermediate maps, with only the original surface and the final flat map remaining the same. However, it is instructive to neuroscientific researchers to understand the deformations and curvature changes that the original surface undergoes to obtain the final quasi-conformal flat map.

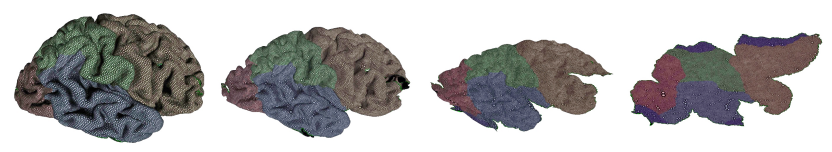

Figure 4: Morphing the Cerebral Cortex onto the Euclidean Flat Map (see color plate). Note that the quasi-conformal flat map is not computed via this morphing process. A movie from a user session is also provided.

\subsection{Interacting with Regions of Interest}

Flat maps of cortical surfaces allow the complex geometry of the brain to be visualized all at once. Although interesting as a visualization tool, neuroscientists want to be able to obtain meaningful information from these flat maps. Interaction between flat maps and $3 \mathrm{D}$ surfaces is needed as a means of being able to understand map distortions, for locating of regions of interest including functional activations and for being able to compare similarities and differences between individuals. With this in mind, our group is developing other visualization tools to gain a greater understanding of the changes the cortical surface undergoes during the flattening process.

Surface area and geodesic distances on the brain are not preserved under any flattening scheme. However, these features are related to neuronal density and functional activation [7]. Thus, it is important for neuroscientists to be able to understand and visualize surface area and the distortion that occurs on a flat map.

We have developed tools using $\mathrm{X}$ windows to allow the user to interact with the flat map and visualize significant properties and relationships between the flat map and cortical surface. One method is to specify a region within the flat map and view its corresponding region in $\mathbb{R}^{3}$. User interaction quickly demonstrated that it was difficult to pinpoint specific regions on the flat map and view their location on a surface mesh in $\mathbb{R}^{3}$ because of the complexity and folds of the brain. A bump mapped image of the flat map was generated to aid in finding particular regions and navigating on the flat map. The software generates the bump mapped flat map by computing a fake diffuse component for each circle using the surface normal in $\mathbb{R}^{3}$. The color for each circle is then scaled based on the diffuse value. Figure 5 (and its corresponding color plate) illustrates the improvement made when using bump map coloring.
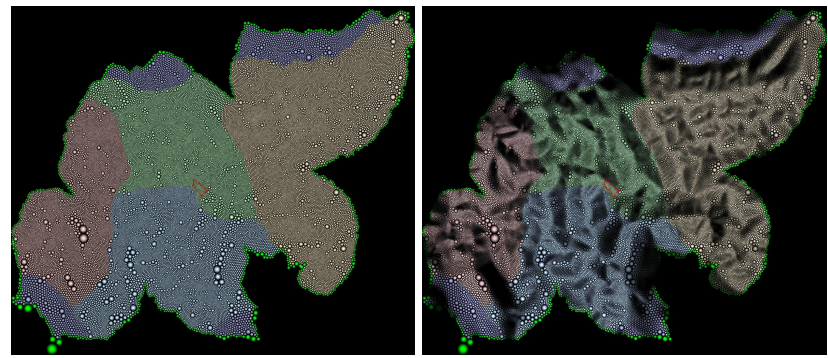

Figure 5: Enhancing the Flat Map. Left: flat map without bump map coloring. Right: bump map coloring assists navigation on the flat map by revealing folds and fissures of the brain (see color plate).

Another feature is that the application calculates the area for the selected region both in $\mathbb{R}^{2}$ and $\mathbb{R}^{3}$ and the results are displayed. A user click on the flat map is translated into a normalized circle point in order to find the closest circle location. After future clicks, the region can be closed and the area is computed for the two dimensional and three dimensional regions using that boundary. A movie from a user session is provided.

For the example given in Figure 6 and its corresponding color plate, the selected region which is indicated by the arrow and outlined (in red on the color plate) has a surface area of $121.82 \mathrm{~mm}^{2}$ in $\mathbb{R}^{2}$. This region underwent considerable area distortion as its original surface area in $\mathbb{R}^{3}$ is $338.89 \mathrm{~mm}^{2}$. Note that the demarcated region on the flattened map is similar in shape to the region on the 3D surface. This is one of the consequences, and advantages, of conformal mapping. From this information, neuroscientists are able to better understand and navigate on the flat map. 

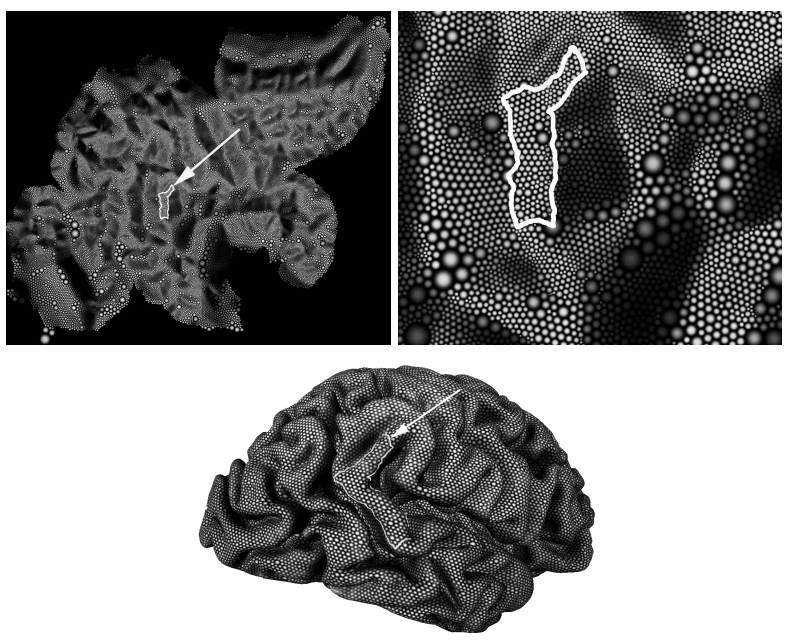

Figure 6: Selecting Regions and Calculating Surface Area. The surface area of the selected region which is indicated by the arrow and outlined (in red on the color plate) is $121.82 \mathrm{~mm}^{2}$ on the flat map and the corresponding area in $\mathbb{R}^{3}$ is $338.89 \mathrm{~mm}^{2}$. Notice that the shape of the region is similar on the cortical surface and flat map. This is one of the advantages of conformal mapping. A movie from a user session is also provided.

\section{Conclusions and Future Work}

One of the goals of neuroscientific researchers is to be able to better understand the functional processing of the human brain and to be able to make conclusions regarding individual differences in functional organization. This can lead to better diagnostic tools for detecting diseases earlier as well as treating brain disorders in new ways. Brain atlases and brain maps can assist in this regard and cortical flat maps are the first step in this process.

These visualization tools represent an initial attempt at being able to interact with cortical flat maps. We are working with neuroscientists to extend the capabilities of these tools by including features such as drawing directly on the mesh in $\mathbb{R}^{3}$ and region to region comparison. The region selecting algorithm can also be enhanced to calculate geodesics on the surface of the brain so the shortest path between two points can be computed and displayed. Extracting and conveying pertinent information from these maps will allow brain maps to be compared and morphed to a template so significant locations, metrics and statistics can be computed. One researcher commented that being able to interactively control the degree of flattening while allowing 3D rotations is quite powerful. Conformal flat maps of cortical surfaces and interaction with them will lead us to better localize functional regions of activation in normal subjects and in patients with hereditary or other diseases.

\section{Acknowledgments}

This work is supported in part by NSF grant 0083898, FSU 2000 Planning grant and NIH Human Brain Project grant MH57180. The authors would like to acknowledge the following people: D.W. Sumners and P. Bowers, Dept. of Mathematics, Florida State U.; K. Stephenson, Dept. of Mathematics, U. of Tennessee, Knoxville; K. Rehm, K. Schaper, J. Stern and D. Rottenberg, VA Medical Center and Depts. of Neurology and Radiology, U. of Minnesota, Minneapolis. D. Van Essen, H. Drury and J. Dickson, from Washington U. School of Medicine, St. Louis, are also gratefully acknowledged for providing the Visible Man data.

\section{References}

[1] L. V. Ahlfors. Complex Analysis. McGraw-Hill Book Company, New York, 1966.

[2] A. F. Beardon and K. Stephenson. The uniformization theorem for circle packings. Indiana Univ. Math. J., 39:13831425, 1990.

[3] B. Fischl, M. I. Sereno, and A. M. Dale. Cortical surfacebased analysis II: Inflation, flattening, and a surface-based coordinate system. Neuroimage, 9:179-194, 1999.

[4] M. K. Hurdal, P. L. Bowers, K. Stephenson, D. W. L. Sumners, K. Rehm, K. Schaper, and D. A. Rottenberg. Quasiconformally flat mapping the human cerebellum. In C. Taylor and A. Colchester, editors, Medical Image Computing and Computer-Assisted Intervention - MICCAI'99, volume 1679 of Lecture Notes in Computer Science, pages 279-286. Springer, Berlin, 1999.

[5] M. K. Hurdal, K. Stephenson, P. Bowers, D. W. L. Sumners, and D. A. Rottenberg. Cortical surface flattening: A quasiconformal approach using circle packings. Neuroimage, submitted. Available as FSU Mathematics Department Report FSU01-14.

[6] International Neuroimaging Consortium (INC), 2001. URL: http://www.neurovia.umn.edu/incweb.

[7] E. R. Kandel, J. H. Schwartz, and T. M. Jessell. Principles of Neural Science. Appleton \& Lange, Norwalk, CT, 1991.

[8] W. E. Lorensen and H.E. Cline. Marching cubes: A high resolution 3D surface construction algorithm. Computer Graphics, 21:163-169, 1987.

[9] D. L. Pham and J. L. Prince. Adaptive fuzzy segmentation of magnetic resonance images. IEEE Trans. on Medical Imaging, 18:737-752, 1999.

[10] B. Rodin and D. Sullivan. The convergence of circle packings to the Riemann mapping. J. Differential Geometry, 26:349$360,1987$.

[11] J. D. Schmahmann, J. Doyon, A. W. Toga, M Petrides, and A. Evans. MRI Atlas of the Human Cerebellum. Academic Press, San Diego, 2000.

[12] J. G. Sled, A. P. Zijdenbos, and A. C. Evans. A nonparametric method for automatic correction of intensity nonuniformity in MRI data. IEEE Trans. on Medical Imaging, 17:87-97, 1998.

[13] K. Stephenson. Approximation of conformal structures via circle packing. In N. Papamichael, St. Ruscheweyh, and E. B. Saff, editors, Computational Methods and Function Theory 1997, Proceedings of the Third CMFT Conference, volume 11, pages 551-582. World Scientific, 1999.

[14] The Human Brain Project, $2001 . \quad$ URL: http://www.nimh.nih.gov/neuroinformatics/index.cfm.

[15] The National Library of Medicine. The Visible Human Project, 2000. URL: http://www.nlm.nih.gov/research/visible/.

[16] A. W. Toga and J. C. Mazziotta, editors. Brain Mapping: The Methods. Academic Press, Inc., San Diego, 1996.

[17] D. C. Van Essen and H. A. Drury. Structural and functional analyses of human cerebral cortex using a surface-based atlas. The Journal of Neuroscience, 17:7079-7102, 1997. 

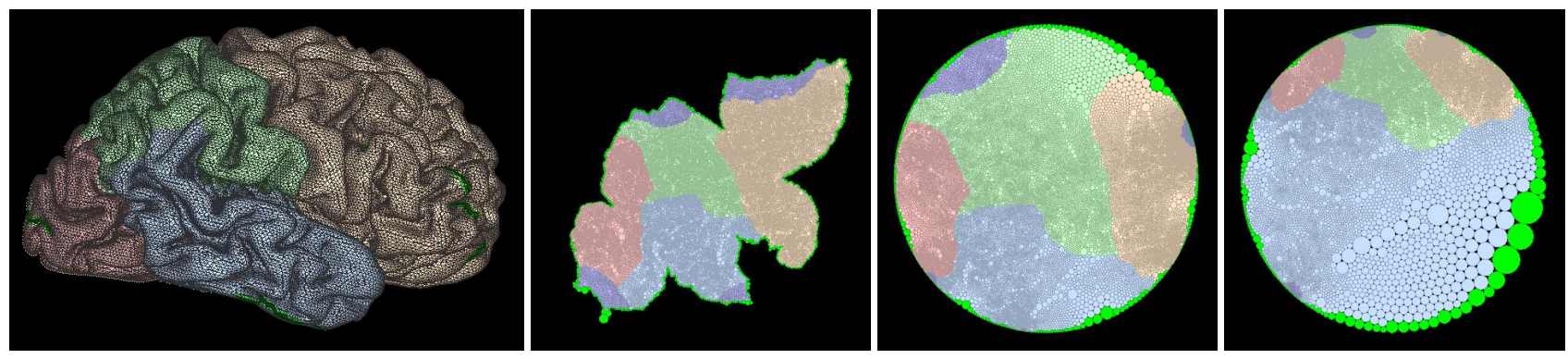

Color Plate of Figure 3: The Human Cerebral Cortex. Left to right: the cerebrum; Euclidean flat map; hyperbolic flat map; hyperbolic map with transformation applied. Colors correspond to various anatomical lobes.
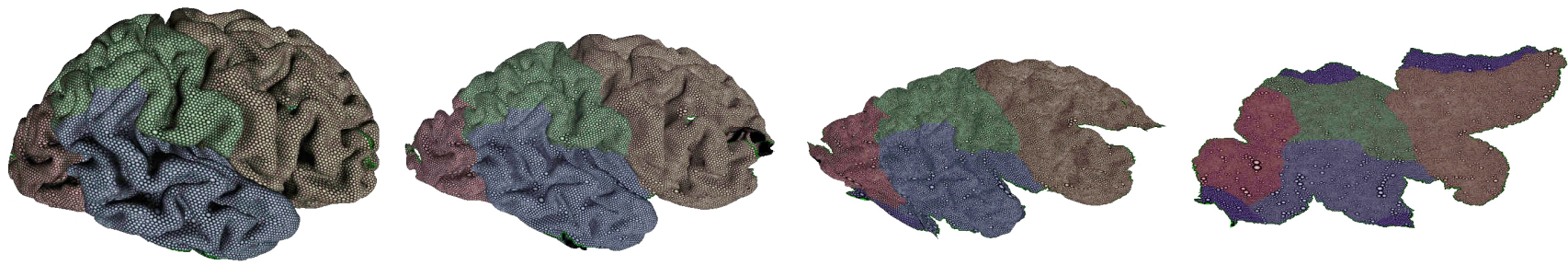

Color Plate of Figure 4: Morphing the Cerebral Cortex onto the Euclidean Flat Map. Note that the quasi-conformal flat map is not computed via this morphing process. A movie from a user session is also provided.
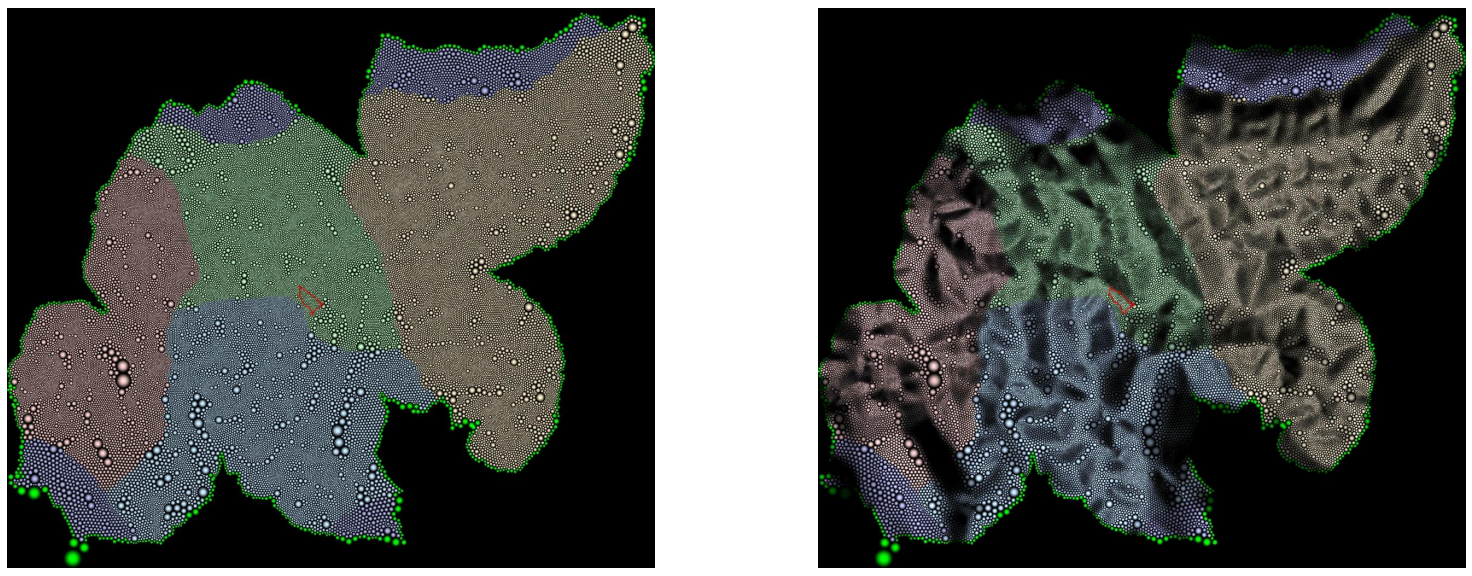

Color Plate of Figure 5: Enhancing the Flat Map. Left: flat map without bump map coloring. Right: bump map coloring assists navigation on the flat map by revealing folds and fissures of the brain.
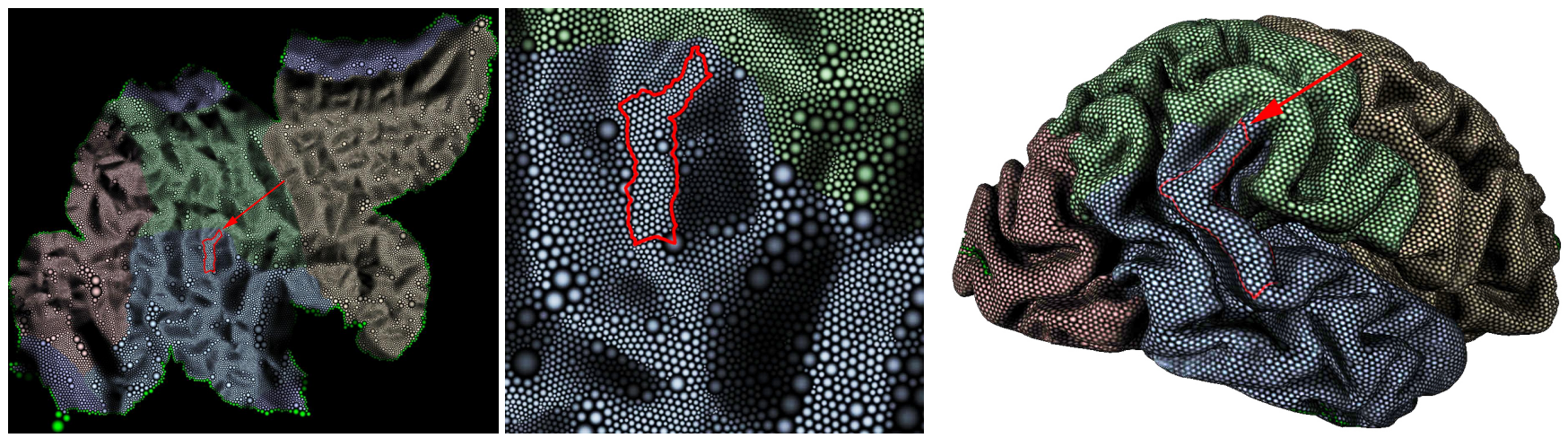

Color Plate of Figure 6: Selecting Regions and Calculating Surface Area. The surface area of the selected region which is indicated by the arrow and outlined (in red on the color plate) is $121.82 \mathrm{~mm}^{2}$ on the flat map and the corresponding area in $\mathbb{R}^{3}$ is $338.89 \mathrm{~mm}^{2}$. Notice that the shape of the region is similar on the cortical surface and flat map. This is one of the advantages of conformal mapping. A movie from a user session is also provided. 